\title{
Optical scattering noise in high $Q$ fiber ring resonators and its effect on optoelectronic oscillator phase noise
}

\author{
Khaldoun Saleh, ${ }^{1,2,3}$ Pierre-Henri Merrer, ${ }^{1,2}$ Olivier Llopis, ${ }^{1,2}$ and Gilles Cibiel ${ }^{3}$ \\ ${ }^{1}$ CNRS; LAAS, 7 Avenue du Colonel Roche, F-31077 Toulouse, France \\ ${ }^{2}$ Université de Toulouse; UPS, F-31077 Toulouse, France \\ ${ }^{3}$ CNES, 18 Avenue Edouard Belin, F-31401 Toulouse, France \\ *Corresponding author: ksaleh@laas.fr
}

Received October 24, 2011; revised December 2, 2011; accepted December 12, 2011; posted December 14, 2011 (Doc. ID 156944); published February 8, 2012

\begin{abstract}
Nonlinear phenomena occurring in an optical fiber ring resonator featuring ultrahigh $Q$ factor are experimentally studied. The laser is locked onto the resonator, and the optical power induced in the resonator is controlled. The onset of the first stimulated Brillouin scattering wave occurs at an optical input power as low as $-9 \mathrm{dBm}$ in these resonators. When the resonator is used as the frequency reference device in an optoelectronic oscillator (OEO), it has been found that these parasitic signals mix with the OEO signal and degrade its phase noise. More than $20 \mathrm{~dB}$ improvement of the OEO phase noise has been demonstrated by limiting these nonlinear optical effects. (C) 2012 Optical Society of America
\end{abstract}

OCIS codes: $250.0250,190.4360,230.5750,230.4910,290.5830,290.5870$.

Presently, high spectral purity microwave signal generation in both optical and electrical domains is usually performed using optical delay lines and the optoelectronic oscillator (OEO) approach [1]. Although delay line based OEOs are easy to use and relatively well known, they remain bulky, their thermal stabilization is difficult, and they produce spurious modes that need complex configurations to be reduced. An alternative solution, instead of using delay lines, is the use of optical resonators featuring ultrahigh optical quality factors $\left(Q_{\mathrm{opt}}>10^{9}\right)$ and relatively low dimensions. Whispering gallery mode resonances in disk or sphere resonators may lead to such $Q$ factors [2]. However, the use of these 3D shape resonators in a system is delicate, because of the mechanical difficulty in setting up a reliable coupling with a light source. Another approach is to take advantage of low losses in optical fibers to realize high $Q$ fiber ring resonators (FRRs). To improve the performance of FRR OEOs, the first investigations have been focused on the resonator optimal coupling and on its thermal stability [3]. However, with regard to the studies done on OEO phase noise improvement, a relatively high $1 /$ f noise component in the phase noise spectrum still exists. In OEO systems, this $1 / \mathrm{f}$ noise may result from the $1 / \mathrm{f}$ noise of active devices, which is folded onto the generated radio frequency (RF) carrier by the system nonlinearities, or it may be due to the resonator intrinsic frequency fluctuations. An example of the first process is the amplitude and frequency noise demodulation of the laser used in the OEO setup. Up to this moment, most of our work has been focused in modeling this behavior. However, we have recently found that a different process was responsible for most of the $1 / f$ phase noise observed in our OEO: the nonlinear optical scattering mechanisms inside the high $Q$ fiber ring resonator.

In this Letter, we will first describe the principle of OEO based on high $Q$ FRR. We will then present experimental investigations on optical scattering mechanisms due to nonlinear optical phenomena occurring inside the resonator, especially the stimulated Brillouin scattering (SBS), and their effect on the OEO phase noise.

The optical FRR, depicted in Fig. 1, is realized using two low loss directional couplers linked with a singlemode polarization-maintaining fiber. The total loop length in our case is $20 \mathrm{~m}$.

Resonances occur if the total integrated phase shift of the incident laser pump wave $\left(\lambda_{\text {laser }} \sim 1.55 \mu \mathrm{m}\right)$ around the resonator ring is an integer multiple of $2 \pi \mathrm{rad}$. This resonator is thus characterized by a single transverse resonant frequency comb with a free spectral range (FSR) related to the fiber length $L$ and its refractive index $n$ : $\mathrm{FSR}=c /(n . L)(\sim 10 \mathrm{MHz}$ in our case $)$. If the coupling factor is chosen small enough $\left(k_{1} \sim k_{2} \sim 1 \%\right)$ and the residual losses of the resonator elements (coupler additional losses, fiber linear losses, and splice losses) are sufficiently low, the resonator will then feature a high optical $Q_{\text {opt }}$ factor equivalent to a long delay line. This being said, the photons inside the resonator will encounter an optical delay $\tau$ as if they were traveling into a given equivalent optical fiber length in single-pass geometry. This equivalent length is given by

$$
L_{\mathrm{eq}}=\frac{Q_{\mathrm{opt}} \cdot c}{n \cdot \pi \cdot f_{\mathrm{opt}}},
$$

where $f_{\text {opt }}$ is the light frequency $\left(193 \times 10^{12} \mathrm{~Hz}\right)$.

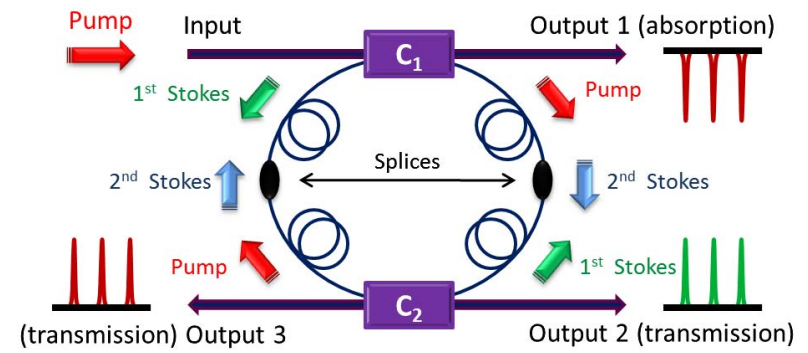

Fig. 1. (Color online) Double directional coupler $20 \mathrm{~m}$ long FRR. 
For example, our $20 \mathrm{~m}$ long FRR with a $Q_{\text {opt }}$ of $3.5 \times 10^{9}$ (accurately measured using the method described in [4]) is equivalent to a $1180 \mathrm{~m}$ long optical delay line. Also, the high $Q_{\text {opt }}$ factor leads to a significant circulating power inside the cavity [5] $\left(P_{\text {cavity }} / P_{\text {in }} \sim 54\right)$, which in turn will reduce the thresholds of many nonlinear optical effects, as we will see later in this Letter.

The $10 \mathrm{GHz}$ OEO stabilized on this FRR is schematically described in Fig. 2 and has been fully explained in [4]. The laser used in this OEO is a Koheras laser with a very narrow linewidth $(\sim 1 \mathrm{kHz})$ and is stabilized on one of the ring resonances using a low frequency PoundDrever-Hall feedback loop. The microwave oscillation is then maintained by another high frequency loop including an amplifier, a phase shifter, and a microwave filter.

In optical fibers many scattering phenomena may occur. Some of them are passive, like Fresnel reflections at the fiber end faces, and some others are active, like Rayleigh, Brillouin, and Raman scattering, which feature two scattering modes each: spontaneous and stimulated scattering.

Theoretically predicted by Brillouin in 1922 [] ], SBS in optical fibers was first observed in 1964 and has been extensively studied since then [ $\underline{7}-10]$. It is caused by the interaction between a light wave and an acoustic wave already existing at the optical medium thermal equilibrium. This interaction creates a scattered wave called a Stokes wave; in this case we are in the spontaneous Brillouin scattering regime. In optical fibers, the backward scattered Stokes wave is privileged and is frequency downshifted from that of the pump incident light by an amount $\nu_{B}$ set by the nonlinear medium characteristics $\left(\nu_{B} \sim 11 \mathrm{GHz}\right.$ in silica fibers [8]). However, a very weak scattered wave called an anti-Stokes wave, travelling in the same direction of the pump and upshifted in frequency by $\nu_{B}$, can be also generated.

At a critical incident light wave power, called the stimulated Brillouin scattering threshold (SBST), the interference between the Stokes wave and the incident light wave amplifies the acoustic wave in a process called electrostriction. Thus, the scattered wave will become more intense and reinforce the interference and so on; we are now in the stimulated regime. When the Stokes power exceeds the medium SBST, it creates a second order Stokes wave downshifted in frequency from the first Stokes wave by $\nu_{B}$, and thus by $2 \nu_{B}$ from the incident light wave.

Our aim here is to study the effects of the Brillouin scattering and other nonlinear phenomena generated

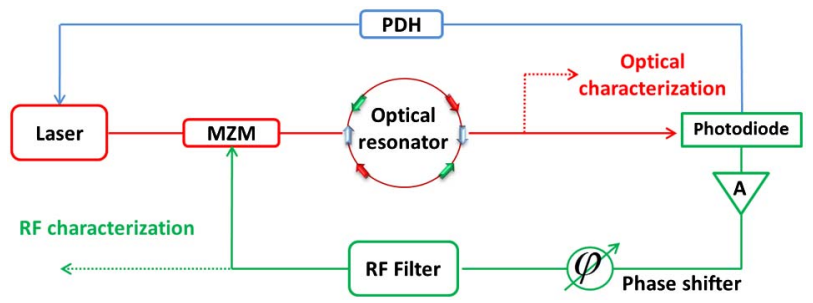

Fig. 2. (Color online) Optoelectronic oscillator at $10 \mathrm{GHz}$. PDH, Pound-Drever-Hall; MZM, Mach-Zehnder modulator; A, RF amplifier.

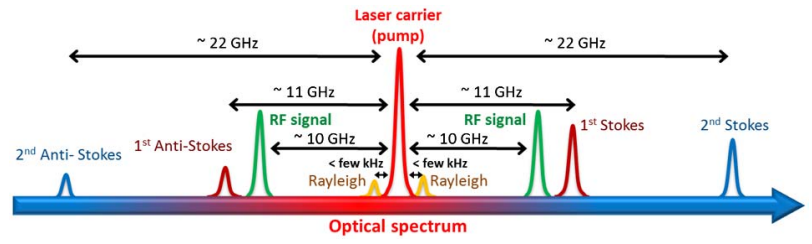

Fig. 3. (Color online) Illustration of the optical spectrum while using the OEO setup in the presence of the first and second Stokes and the Rayleigh scattering.

inside the resonator on the OEO frequency stability. Further information on these optical phenomena can be found in [7-11].

The SBST in an optical fiber in single-pass geometry will be drastically reduced if the same fiber length is used in resonator geometry, depending on the resonator quality factor [9]. Unfortunately, in our $10 \mathrm{GHz}$ OEO, this SBST reduction will result in many higher order Brillouin lines in the optical spectrum, (the seventh Brillouin line has been observed at $10 \mathrm{dBm}$ input power), which will mix with the OEO fundamental RF oscillation and degrade its phase noise (see Fig. 3).

The first and second Stokes wave powers have been measured at the resonator's 2nd and third outputs, respectively, as a function of the laser (pump) input power in the optical domain. We use an Agilent HP 86142A optical spectrum analyzer (OSA) with $0.06 \mathrm{~nm}$ resolution and $\pm 1 \mathrm{~dB}$ amplitude precision. The results are depicted in Fig. 4 . Under the first Stokes SBST $(\sim-9 \mathrm{dBm})$, when there are no additional nonlinear losses, the ring resonator total loss between the input port and the third output port is about $3.7 \mathrm{~dB}$. Above the first SBST, the laser pump at the third output starts to saturate, and additional pumped energy inside the resonator is converted into first Stokes wave energy with a conversion ratio that reaches $69 \%$ at $-2 \mathrm{dBm}$ input power. Above the second SBST $(\sim-2 \mathrm{dBm})$, the first Stokes wave starts to saturate in its turn when the second Stokes wave, spaced by $10.8 \mathrm{GHz}$ from the first Stokes wave, starts to be in a stimulated regime. Because of the OSA's limited resolution, the second Stokes wave power measurements were inaccurate for laser input power below $-2 \mathrm{dBm}$.

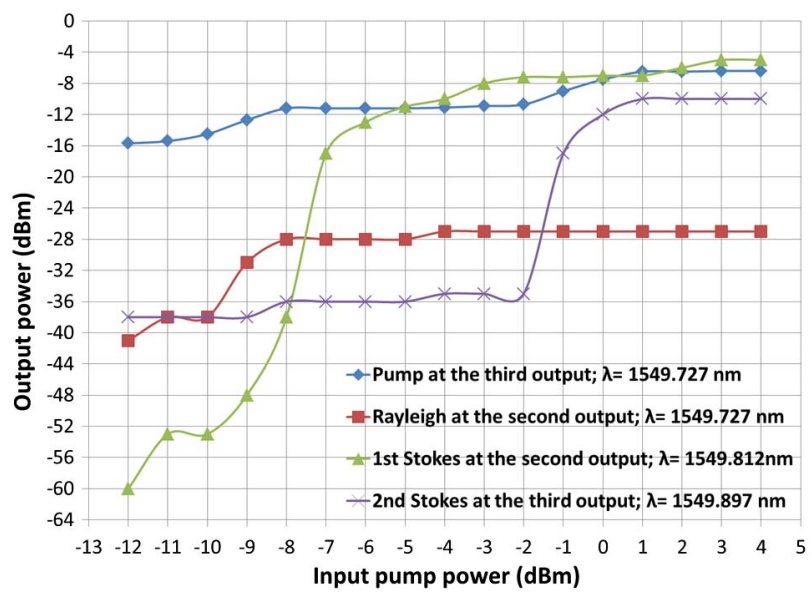

Fig. 4. (Color online) Resonator output laser pump, Rayleigh, and first and second Stokes powers as a function of the laser pump input power. 


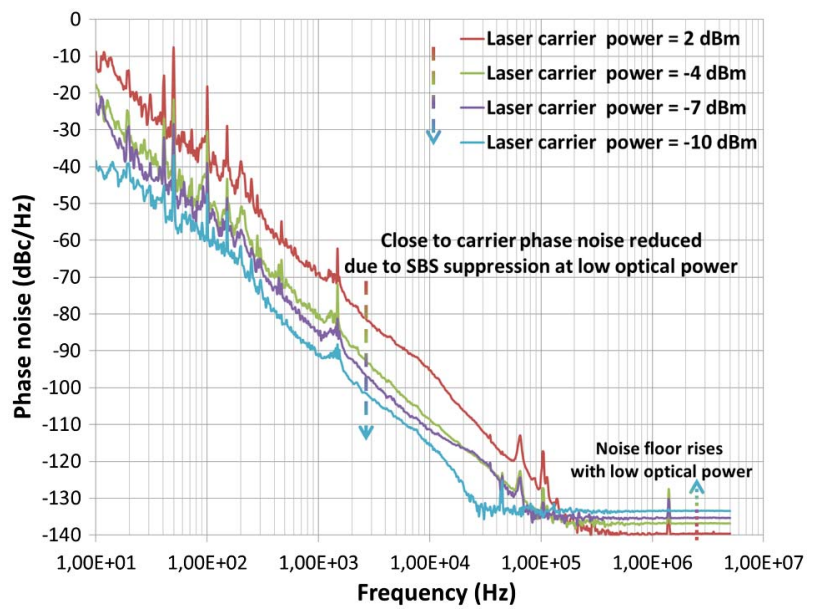

Fig. 5. (Color online) $20 \mathrm{~m}$ long, fiber ring resonator based $10 \mathrm{GHz}$ OEO phase noise reduction due to SBS suppression at low optical carrier power.

The Rayleigh scattering power has been also measured at the second output. We have found that its power increases with the laser input power but remains constant after the Brillouin scattering process starts to be stimulated. Such a Rayleigh-Brillouin scattering behavior has been already thoroughly studied in single-pass geometry optical fibers $[10,11]$. It has been noticed in [10] that at power levels above SBST, the SBS draws power away from the scattering process, thereby clamping the stimulated Rayleigh scattering gain, and moreover, both mechanisms can contribute to the noise induced on signals generated by delay line OEOs.

Adding the weak (frequency upshifted) anti-Stokes waves and the close to optical carrier (less than a few kilohertz $[10,11])$ Rayleigh scattering to the Brillouin Stokes waves generated around the optical carrier (Fig. 3), we expect that our OEO RF signal at $\sim 10 \mathrm{GHz}$ will be affected by the presence of all these nonlinear optical phenomena and, more precisely, by the nonlinear noise conversion processes between all these optical carriers. One of these processes is the DC noise detection by the photodiode of all these lines, which are again folded onto the RF carrier either by the photodiode nonlinearity or inside the resonator through interference processes. Therefore, the OEO RF carrier phase noise has been carefully measured with an Agilent E5052 B signal source analyzer at different laser input power levels. The experimental results are depicted in Fig. $\underline{5}$.

The $1 / \mathrm{f}$ phase noise observed near the carrier is strongly dependent on the laser power. We can clearly notice a significant enhancement of the close to carrier OEO phase noise at low optical input power, from $-10 \mathrm{dBc} / \mathrm{Hz}$ at $10 \mathrm{~Hz}$ offset frequency and $2 \mathrm{dBm}$ (above the second SBST) input laser carrier power to $-40 \mathrm{dBc} / \mathrm{Hz}$ at $-10 \mathrm{dBm}$ (under the first SBST). An increment in the noise floor can be noticed when the carrier power decreases, because it is directly linked to the noise to carrier ratio. However, in the OEO case, the close to carrier performance is a more sensitive parameter for most applications (radar, time and frequency references, etc.).

These results clearly demonstrate that optical power in the resonator has to be maintained below the first SBST power to get an adequately low phase noise in an FRR OEO. Our computation reveals that the phase noise was not limited by RF amplifier noise and that it is probably still limited by a contribution of the Rayleigh scattering noise. When the optical power is reduced, SBS is reduced. But as has been noticed in [10], the Rayleigh noise spectrum becomes broader at low power, and thus it affects the phase noise of the RF signal at higher offset frequencies.

In this Letter, the onset of nonlinear optical effects in a optical fiber ring resonator have been studied, especially stimulated Brillouin scattering. We have experimentally demonstrated the correlation between these phenomena and the spectral purity of a $10 \mathrm{GHz}$ microwave signal generated by an optoelectronic oscillator based on this resonator. The setup of an oscillation with a laser carrier power below the first stimulated Brillouin scattering threshold results in an improved phase noise performance of the oscillator.

This study is part of a French National Research Agency (ANR) project ORA (2010 BLAN 0312 03).

\section{References}

1. X. S. Yao and L. Maleki, in 2004 IEEE MTT-S International Microwave Symposium Digest (IEEE, 2004), pp. 287-290.

2. A. A. Savchenkov, V. S. Ilchenko, A. B. Matsko, and L. Maleki, Phys. Rev. A 70, 05804 (2004).

3. K. Saleh, A. Bouchier, P. H. Merrer, O. Llopis, and G. Cibiel, Proc. SPIE 7936, 79360A (2011).

4. P. H. Merrer, A. Bouchier, H. Brahimi, O. Llopis, and G. Cibiel, in Proceedings of the 2009 Joint Meeting of the European Frequency and Time Forum (EFTF) and the IEEE International Frequency Control Symposium (FCS) (IEEE, 2009), pp. 866-869.

5. F. Zhang and J. Lit, J. Opt. Soc. Am. A 5, 1347 (1988).

6. L. Brillouin, Ann. Phys. 17, 88 (1922).

7. A. Kobyakov, M. Sauer, and D. Chowdhury, Adv. Opt. Photon. 2, 1 (2010).

8. G. P. Agrawal, Nonlinear Fiber Optics, 4th ed. (Academic, 2007), Chap. 9.

9. L. F. Stokes, M. Chodorow, and H. J. Shaw, Opt. Lett. 7, 509 (1982).

10. O. Okusaga, J. Cahill, W. Zhou, A. Docherty, G. M. Carter, and C. R. Menyuk, in Proceedings of the 2011 Joint Conference of the IEEE International Frequency Control and the European Frequency and Time Forum (FCS) (IEEE, 2011), pp. 1-6.

11. T. Zhu, X. Bao, L. Chen, H. Liang, and Y. Dong, Opt. Express 18, 22958 (2010). 